\title{
La apertura comercial ¿ha creado paraísos de contaminadores en América Latina?
}

\section{Rhys Jenkins}

School of Development Studies Universidad de East Anglia, Norwich, Reino Unido R.O.Jenkins@uea.ac.uk
El presente artículo analiza si los recientes procesos de apertura comercial en Argentina, Brasil y México han provocado un aumento de la transferencia de actividades contaminantes hacia América Latina. Utilizando un enfoque basado en el contenido factorial del comercio, demuestra que antes de esa liberalización los tres países se especializaban en industrias altamente contaminantes. Después de la apertura, en Argentina y Brasil se incrementó la especialización en industrias de esa índole, mientras que en México se siguió la trayectoria opuesta. Se sugiere que estas diferencias son el resultado de la estructura de protección anterior al período de apertura y de la aplicación cada vez más estricta de las leyes ambientales en México durante el decenio de 1990. 


\section{I}

\section{Introducción}

El temor de que la apertura comercial sea incompatible con el desarrollo sostenible fue uno de los principales argumentos esgrimidos por los manifestantes que protestaban ante la reunión de la Organización Mundial del Comercio (омс) en Seattle, Estados Unidos, en 1999. Uno de los motivos esenciales de preocupación para los ambientalistas es la creencia de que los países en desarrollo se convertirán en "paraísos de contaminadores" que atraerán a las industrias "sucias" gracias a sus estándares ambientales menos estrictos (Mabey y McNally, 1999). Los partidarios de la apertura comercial, por su parte, aducen que este proceso tendrá consecuencias ambientales beneficiosas, tanto a nivel mundial como en los países en desarrollo (Esty, 1994).

En varios modelos teóricos de comercio norte-sur se predice que la normativa ambiental menos estricta provocará un incremento de la producción contaminante en el hemisferio sur cuando se liberalice el comercio. ${ }^{1}$ Dado que una normativa ambiental menos exigente conlleva costos relativos más bajos para las industrias muy contaminantes, en el hemisferio sur existiría una ventaja comparativa en la producción de bienes "sucios". Por el contrario, el hemisferio norte, caracterizado por normas ambientales más exigentes, tendería a especializarse en productos "limpios". 2

Uno de los supuestos clave de estos modelos es que la diferencia principal entre los hemisferios norte y sur es el nivel de regulación ambiental. Quienes creen que la apertura comercial puede tener efectos positivos en el medio ambiente han señalado que, en general, los costos de control ambiental de la industria manufacturera son bajos, y que hay factores distintos de los ambientales que tienen más peso como determinantes de ventajas comparativas (Dean, 1992). En este caso, es bastante probable que en un país en desarrollo caracterizado por un sistema de control ambiental menos estricto exista, de todas formas, una ventaja comparativa en industrias menos contaminan-

$\square$ El autor expresa su agradecimiento a un juez anónimo por sus comentarios a una versión anterior de este mismo artículo.

${ }^{1}$ Véase, por ejemplo, Baumol y Oates (1988) y Copeland y Taylor (1994).

2 En este contexto, los términos "sucio" y "limpio" indican, respectivamente, si las industrias que producen un bien determinado son altamente contaminantes o si no lo son. tes. Cuando hay correlación entre la intensidad con que se utiliza el capital y la intensidad de contaminación, los países en los que exista una ventaja comparativa en industrias con uso intensivo de mano de obra obtendrán beneficios ambientales de una especialización acorde a esta ventaja. De hecho, la contaminación tenderá a aumentar en el hemisferio norte dada su especialización en industrias con uso intensivo de capital, y se reducirá en el hemisferio sur (Antweiler, Copeland y Taylor, 1998). Este punto de vista está relacionado con el concepto de que la estructura de protección de los países en desarrollo tiene un "sesgo sucio". En otras palabras, se sugiere que, en regímenes de sustitución de importaciones, las industrias altamente contaminantes tienden a recibir más protección que las menos contaminantes (Birdsall y Wheeler, 1992).

El efecto total de la apertura comercial en el medio ambiente depende no sólo de la composición de la producción, sino también de su volumen y de la tecnología (Grossman y Krueger, 1992). Pero en la medida en que conduzca al crecimiento de la producción industrial, tenderá a provocar un incremento de la contaminación. Por otra parte, en los países en los que la reforma comercial lleve a la adopción de procesos productivos menos contaminantes - por ejemplo, gracias a la disponibilidad creciente y el costo reducido de las tecnologías importadas, o a la exigencia de que estas tecnologías se apliquen al producir para mercados de exportación - la contaminación por unidad de producción tenderá a disminuir. Por ese motivo, para evaluar el efecto global de esa apertura en el medio ambiente de un país sería necesario considerar tres tipos de efectos: de escala, de composición y de proceso tecnológico. $^{3}$

El presente artículo tiene un propósito más restringido. En tanto que el efecto de escala se traduce claramente en un incremento de la contaminación, ${ }^{4}$ y que

\footnotetext{
${ }^{3}$ En Beghin y Potier (1997) se pasa revista a los estudios sobre las consecuencias de la apertura comercial en la contaminación procedente del sector manufacturero, teniendo en cuenta los tres efectos señalados.

${ }^{4}$ Existe, evidentemente, el argumento de la curva ambiental de Kuznets, según el cual, cuando se superan determinados niveles de ingreso por habitante, los niveles de contaminación tienden a descender. Sin embargo, esto sucede cuando los efectos de composición y de proceso tecnológico sobrepasan el efecto de escala.
} 
en general se considera que el efecto de proceso tecnológico la reduce, el efecto de composición es ambiguo. En la hipótesis de los paraísos de contaminadores se sugiere que, al liberalizar el comercio, en el hemisferio sur se tenderá a la especialización en industrias contaminantes, dada su legislación ambiental poco exigente. ${ }^{5}$ Para expresarlo de otra forma, se afirma que los efectos de composición asociados a la apertura del comercio tenderán a incrementar la contaminación en el hemisferio sur. Dado que este artículo no se ocupa de las consecuencias de esa apertura en la escala de producción industrial ni en el proceso tecnológico, no puede determinar si ella incrementa o reduce el nivel de contaminación industrial en el ámbito local. Lo que pretende es analizar si la apertura comercial ha producido mayor o menor especialización en industrias altamente contaminantes y si, en consecuencia, el efecto de composición de esa apertura ha sido positivo o negativo para el medio ambiente.

La apertura que ha tenido lugar en América Latina desde la segunda mitad de la década de 1980 hasta la fecha indica que esta región es apropiada para analizar las afirmaciones y refutaciones acerca de las con- secuencias ambientales de esa liberalización. Dado que tres cuartas partes de la producción industrial de la región corresponden a tres países - Argentina, Brasil y México-, se decidió concentrar el estudio en este grupo. En el artículo se analiza si la apertura del comercio ha estado relacionada con un incremento de la especialización de los países latinoamericanos en industrias muy contaminantes.

A continuación, en la sección II se describen los grandes cambios de política económica que tuvieron lugar en Argentina, Brasil y México a partir de la segunda mitad de la década de 1980. La sección III contiene un resumen crítico de diversos estudios anteriores sobre la relación del comercio con la contaminación en América Latina. La sección IV presenta una visión alternativa de esta relación, basada en un modelo de comercio en el cual la contaminación se considera un factor de producción más. En la parte empírica del artículo (sección V) se estudia la "intensidad de contaminación" relativa de exportaciones e importaciones en los tres países señalados, así como los cambios de estas variables a partir de la apertura, y en la sección VI se ofrecen algunas conclusiones.

\section{II}

\section{Apertura en América Latina}

En el período transcurrido desde la mitad de la década de 1980 hasta la fecha, en las economías latinoamericanas se ha experimentado una amplia liberalización. Aunque en este proceso han participado muchos otros elementos — como privatizaciones, desregulaciones financieras, reformas fiscales y modificaciones de los mercados laborales_- los cambios más rápidos e impresionantes se han dado en la apertura de las

\footnotetext{
${ }^{5}$ En Jensen (1996) se analizan las distintas formulaciones de la hipótesis de los paraísos de contaminadores. Este autor señala que en varios estudios se hace hincapié en que usar deliberadamente una normativa ambiental débil para atraer capital equivale a implantar un paraíso de contaminación. Sin embargo, esta definición, que depende de las motivaciones de los responsables políticos, no puede comprobarse empíricamente mediante el análisis de la estructura del comercio internacional. Jensen ofrece una definición alternativa: dadas las diferencias políticas en cuanto a los niveles aceptables de emisiones, algunos países pueden obtener una ventaja comparativa al producir bienes con procesos industriales muy contaminantes (Jensen, 1996, p. 320). Esta es la interpretación que se da a la hipótesis de los paraísos de contaminadores en este artículo. También es el sentido que se da al término en el conocido artículo de Birdsall y Wheeler (1992).
}

economías de la región mediante la liberalización del comercio y la modificación de las políticas sobre inversión extranjera directa (IED).

De los tres países latinoamericanos más grandes, México fue el primero en abrir su economía, a mediados de la década de 1980. Luego siguieron Argentina y Brasil, y una larga lista de países en los que la apertura comenzó alrededor de $1990{ }^{6}$ La liberalización comercial supuso una reducción del nivel medio de derechos de importación, aranceles más uniformes (menor dispersión), el descenso de la proporción del comercio sujeta a barreras no arancelarias y la reducción de los impuestos a la exportación. Entre mediados del decenio de 1980 y mediados del decenio de 1990 el arancel medio se redujo de $50 \%$ a $14 \%$ en Argentina, de más de

\footnotetext{
${ }^{6}$ Cada autor da una fecha diferente para marcar el inicio de la liberalización comercial en cada país, pero todos ellos coinciden en que las fechas clave aproximadas son 1985 en México y 1990 en Argentina y Brasil. Véase Agosin y Ffrench-Davis (1993), cuadro 1; Edwards (1995), cuadro 1-1; Burki y Perry (1997).
} 
$80 \%$ a $13 \%$ en Brasil y de más de $40 \%$ a $14 \%$ en México (Burki y Perry, 1997, cuadro 2.2; BID, 1997, gráfico 17). A principios de la década de 1990, la proporción de partidas sujetas a barreras no arancelarias era inferior al $4 \%$ en México, $1,5 \%$ en Brasil y $0,2 \%$ en Argentina (ibíd.).

A la vez que se desmantelaban las barreras al comercio, en estas economías se flexibilizaron los controles de las inversiones entrantes. Una vez más, México fue el primero en abrir las puertas al capital extranjero. A partir de 1984, el marco regulatorio se hizo menos restrictivo; en 1989, mediante un nuevo conjunto de normas se abrogaron todas las disposiciones anteriores sobre inversión extranjera y se amplió el espectro de posibilidades, admitiendo incluso que la propiedad del capital de las empresas fuera $100 \%$ extranjera. En 1993 se aprobó una nueva ley que consolidaba estos cambios (Ros, Draisma y otros, 1996) y, un año más tarde, el Tratado de Libre Comercio (TLC) de América del Norte abrió aún más la economía mexicana tanto al comercio como a la inversión procedente de los Estados Unidos y Canadá. En Argentina, a partir de 1989 se eliminaron las restricciones a la inversión extranjera en varios sectores, entre ellos la informática, las telecomunicaciones y la electrónica. En 1993 se aprobó la Ley de Inversiones Extranjeras, mediante la cual se abría aún más la economía a los capitales extranjeros y se eliminaban todas las restricciones a las remesas de utilidades (Chudnovsky y López, 1997). Alrededor de 1994, Argentina había desregulado totalmente las inversiones extranjeras (Edwards, 1995, cuadro 7-10). En Brasil el cambio fue menos acelerado que en México y Argentina. De hecho, en la Constitución de 1988 se impusieron más controles a las actividades de las empresas extranjeras. Sin embargo, en la década de 1990 se eliminaron los controles sobre la salida de capitales y se permitió la entrada de empresas extranjeras en el sector de las tecnologías de la información. Mediante las reformas constitucionales de 1993 y las enmiendas subsiguientes, aprobadas en 1995, se liberalizó aún más la política en materia de inversiones extranjeras (Chudnovsky y López, 1997).

Los resultados de la apertura comercial se hicieron ver claramente en los tres países en la década de 1990. El porcentaje del producto interno bruto (PIB) correspondiente al comercio aumentó, primero en México a partir de mediados del decenio de 1980, y a continuación en Argentina y Brasil en la década de 1990 (cuadro 1). De manera análoga, la participación de la inversión extranjera en la formación bruta de capital fijo registró un incremento considerable en el período posterior a las reformas.

CUADRO 1

Argentina, Brasil y México: Comercio ${ }^{\mathrm{a}}$ e inversión extranjera directa (IED) ${ }^{\mathrm{b}}$ como proporción del producto interno bruto (Porcentajes)

\begin{tabular}{lrrrr}
\hline & 1980 & 1990 & 1995 & 2000 \\
\hline Argentina & & & & \\
$\quad$ Comercio & 15,4 & 14,5 & 21,4 & 25,7 \\
IED & 6,9 & 6,4 & 10,8 & 25,6 \\
Brasil & & & & \\
$\quad$ Comercio & 12,9 & 12,6 & 18,2 & 21,9 \\
$\quad$ IED & 7,4 & 8,0 & 6,0 & 33,1 \\
México & & & & \\
$\quad$ Comercio & 27,6 & 32,9 & 46,6 & 77,7 \\
IED & 3,6 & 8,5 & 14,4 & 16,9 \\
\hline
\end{tabular}

Fuente: Para las cifras de comercio: CEPAL (2001), cuadros 58 y 61. Para las cifras de acervo de inversión extranjera directa: UNCTAD (2002), anexo, cuadro B6.

a Por "comercio" se entiende aquí la participación del comercio total (importaciones + exportaciones) en el producto interno bruto.

b Por "IED" se entiende aquí el total de inversión extranjera directa como porcentaje del producto interno bruto.

\section{III}

\section{Estudios anteriores sobre comercio}

\section{y contaminación en América Latina}

Para comprender mejor la relación entre las políticas de comercio y la contaminación industrial en América Latina se podría recurrir a dos tipos de estudios previos. ${ }^{7}$ El primer tipo se ocupa de la intensidad de la contaminación causada por la producción industrial en general, y de su variación de un país a otro y a lo largo del tiempo. El segundo examina de manera más específica el co-

\footnotetext{
${ }^{7}$ En este artículo se considerarán solamente estudios ex post de la estructura comercial. Se han realizado varios intentos de estimar el
} 
mercio, y sobre todo las exportaciones, para determinar el nivel de contaminación que producen y su variación en el tiempo.

En un influyente estudio del primer grupo, realizado por dos economistas del Banco Mundial, se concluyó que los paraísos de contaminadores existen, pero no donde generalmente se les ha buscado; donde se encuentran es en las economías proteccionistas (Birdsall y Wheeler, 1992).

Esta afirmación se basa en la constatación de que, en las economías más abiertas de la región, la tasa de aumento de la contaminación tendía a ser menor que en las economías más proteccionistas.

Un examen crítico de ese estudio, sin embargo, ha señalado varios puntos débiles (Rock, 1996). En primer lugar, sólo considera emisiones tóxicas que no están necesariamente en relación directa con los contaminantes convencionales del aire y el agua. En segundo lugar, mide la apertura comercial sobre la base del Índice de Dollar, que también ha sido cuestionado (Rodrik, 1994). En tercer lugar, Birdsall y Wheeler comparan la tasa de aumento de la intensidad de la contaminación en las economías cerradas y abiertas, y no su nivel absoluto. Y por último, se ha sugerido que el aumento menor de la contaminación en las economías más abiertas puede ser una "creación estadística" ${ }^{8}$ De las propias estimaciones de Rock (no sólo para América Latina) se desprende que la composición de la producción en las economías más abiertas tiende a ser más contaminante.

Según un trabajo más reciente del Banco Mundial (Mani y Wheeler, 1999), la participación de industrias contaminantes en la producción latinoamericana ha aumentado de manera sostenida desde comienzos de la década de 1960, lo que contrasta radicalmente con su disminución en Europa, América del Norte y Japón. Esto significa que en América Latina la producción ha tendido a hacerse más contaminante. De hecho, en un

posible efecto de la liberalización comercial sobre la contaminación mediante modelos de equilibrio general computable, tanto para países individuales como a nivel mundial. Aquí no se examinará este tipo de análisis, ya que tiende a operar con sectores muy amplios y, en todo caso, son estudios ex ante de los efectos predecibles de la liberalización, de modo que los resultados dependen de las características del modelo. Véase una reseña de algunos de estos estudios en Beghin y Potier (1997).

${ }^{8}$ En concreto, se señala que la variable empleada para medir la orientación comercial es una medida de interacción entre la tasa de crecimiento del ingreso per cápita y el llamado Índice de Dollar de la orientación comercial. En Rock (1996) se sugiere que esta variable refleja los efectos del crecimiento, en vez de la orientación comercial. estudio del caso mexicano se señala que, hasta fines de la década de 1980, la toxicidad de la producción aumentó considerablemente (Ten Kate, 1993).

$\mathrm{Si}$ se trata de analizar las consecuencias de la apertura en lo que respecta a la contaminación, estos estudios tienen la importante limitación de abarcar solamente hasta mediados o fines de la década de 1980 o, como mucho, principios del decenio de 1990. Dado que, como se indicó, el proceso de apertura se inició en México sólo a mediados de la década de 1980 y en Argentina y Brasil a principios del decenio de 1990, para evaluar la evolución de la contaminación en este último decenio se necesitan datos más recientes. Una segunda limitación es que todos estos estudios se han concentrado en las emisiones tóxicas que, como ya se señaló, no son necesariamente representativas de todas las formas de contaminación. Por último, como en ellos se considera la producción en su conjunto, las tendencias detectadas no siempre reflejan únicamente los efectos del comercio en la contaminación, sino que pueden responder a otras influencias.

El segundo grupo de estudios, en los que se trata directamente la relación entre el comercio y la contaminación en América Latina, es más apropiado para el análisis de los efectos de la liberalización comercial. Un trabajo realizado por el World Resources Institute estimó la contaminación total atribuible a la producción destinada a la exportación en varios países latinoamericanos, sobre todo a principios de la década de 1990. Asimismo, examinó los cambios en la proporción de la producción destinada a la exportación de varias industrias y llegó a la conclusión tentativa de que los sectores que producen menor contaminación tienden a tener una incidencia levemente mayor en el aumento de las exportaciones que los sectores altamente contaminantes (Runge y otros, 1997). Los datos empleados en los casos de Argentina y Brasil fueron muy limitados, pero en el caso mexicano, la participación de las exportaciones en la producción de las industrias muy contaminantes disminuyó de manera apreciable a comienzos del decenio de 1990.

En un estudio más reciente y detallado de la CEPAL acerca de nueve países latinoamericanos se determinó que, desde principios de la década de 1980, en todos ellos el volumen de las exportaciones de industrias "sucias" tendió a aumentar en términos absolutos. Sin embargo, la participación de estas industrias en las exportaciones disminuyó considerablemente a fines del decenio de 1980 y principios del decenio de 1990 en todos esos países, salvo Brasil (Schaper, 1999). La tendencia reciente, por lo tanto, ha favorecido a las industrias menos contaminantes. 
En otro estudio de la CEPAL sobre ocho de los nueve países considerados por Schaper, el panorama fue más variado: en Brasil, Colombia y Costa Rica aumentó la participación de las exportaciones de manufacturas provenientes de industrias contaminantes (Schatan, 1999); en los otros cinco países, el aumento de la contaminación asociada a las exportaciones se debió al mayor volumen de éstas y no a un cambio en su composición hacia industrias más contaminantes. ${ }^{9}$

Hay también unos pocos estudios de países individuales en los que se ha examinado la relación entre la estructura de las exportaciones y la contaminación. En Argentina, la participación de las industrias altamente contaminantes en las exportaciones que van tanto a los países de la Organización de Cooperación y Desarrollo Económicos (OCDE) como al resto del mundo, disminuyó de 1990 a 1997 (Chudnovsky, Porta y otros, 1996, cuadro V.1; Chudnovsky, Cap, Trigo y Rubin, 1999, cuadros 9 y 10).

En Brasil, la participación de las industrias "sucias" en las exportaciones es relativamente alta. Aumentó sensiblemente en la década de 1980 (Jha, Markandya y Vossenaar, 1999, cuadro 4.3) y siguió siendo particularmente dinámica en la primera mitad del decenio de 1990 (Ferraz y Young, 1999). Una prueba adicional de la especialización del país en industrias relativamente contaminantes es que, de acuerdo con la mayoría de los indicadores de contaminación, en los decenios de 1980 y 1990 la intensidad de contaminación media de las exportaciones superó a la del sector manufacturero en su conjunto (Young, 1998; MмA, 2001). Asimismo, se ha señalado que las exportaciones tienen un componente de contaminación bastante superior al de las importaciones (Young, Lustosa y otros, 2001, cuadro 4.3).

Respecto de México, la inquietud sobre el impacto ambiental del TLC de América del Norte motivó, a principios de la década de 1990, una serie de estudios sobre la estructura de su comercio (Grossman y Krueger, 1992; Low, 1992). Según estos estudios, las exportaciones mexicanas no se concentraron fuertemente en industrias muy contaminantes y las importaciones estadounidenses procedentes de México no respondieron a reducciones de costos en los Estados Unidos. En un

\footnotetext{
${ }^{9}$ Estos dos estudios difieren en la clasificación de las exportaciones - uso de la Clasificación Uniforme para el Comercio Internacional (CUCI) o de la Clasificación Industrial Internacional Uniforme de todas las actividades económicas (CIIU) - ; en el universo considerado - exportaciones totales o exportaciones de manufacturas-, y en el período que abarcan.
}

estudio del período previo a la firma de dicho TLC se encontró que, en términos generales, la apertura comercial en México en el decenio de 1980 y comienzos del decenio de 1990 tuvo efectos positivos para el medio ambiente (Aroche, 2000), mientras que en otro trabajo, posterior a la entrada en vigor del TLC, se concluyó que la estructura de las exportaciones mexicanas había evolucionado hacia una menor intensidad de contaminación (Schatan, 2000).

El panorama que emerge de estos estudios sobre la relación entre el comercio y el medio ambiente en América Latina no parece apoyar la hipótesis de paraísos de contaminadores creados por la transferencia de las actividades productivas contaminantes hacia la región. De hecho, los datos indican que en los últimos años Argentina, México y otros países de la región han tendido más bien a alejarse de la especialización en industrias "sucias".

Sin embargo, tales trabajos tienen limitaciones que obligan a examinar el tema más a fondo. En primer lugar, casi todos los estudios del comercio consideraron exclusivamente las exportaciones. ${ }^{10} \mathrm{Un}$ examen más completo de los efectos de la liberalización comercial en lo que respecta a la especialización en industrias muy contaminantes exige asimismo el análisis del perfil de las importaciones. Por consiguiente, en el presente artículo se tendrá en cuenta la intensidad de contaminación tanto de las importaciones como de las exportaciones. ${ }^{11}$

En segundo lugar, en muchos estudios se hace hincapié en la participación de las industrias altamente contaminantes en las exportaciones de manufacturas desde la región, lo que plantea algunos problemas. ${ }^{12}$ La definición de "industrias sucias" varía de un estudio a otro. Para clasificar las industrias según el nivel de contaminación que producen se utilizan diferentes indicadores. Algunas industrias toman como indicador

\footnotetext{
10 Algunos de los estudios sobre Brasil (Young, 1999; Young, Lustosa y otros, 2001) y un estudio sobre México (Aroche, 2000) son la excepción.

${ }^{11}$ Como señala Aroche (2000), resulta fundamental tener en cuenta las importaciones dado que, de manera implícita, sustituyen a la producción y su contaminación asociada, que se traslada al país productor de los bienes.

${ }^{12}$ Esta variable se utiliza en Schaper (1999); Chudnovsky, Cap y otros (1999); Jha, Markandya y Vossenaar (1999) y Low (1992). En Runge y otros (1997) se examinan las variaciones en la participación de las industrias "sucias" en las exportaciones de América Latina, mientras que en Grossman y Krueger (1992) se emplea como variable dependiente la proporción de las importaciones procedentes de México respecto de la producción, por industria, de los Estados Unidos.
} 
el porcentaje de los costos que se dedica a reducir la contaminación, mientras que otras usan datos de las emisiones. Por añadidura, el umbral para definir a una industria como altamente contaminante es en esencia arbitrario. Se puede evitar la clasificación arbitraria si se emplean coeficientes de emisión para todas las industrias y se aplican a los niveles de exportaciones e importaciones para medir la intensidad media de contaminación del comercio, como se hace en el presente artículo.

Una tercera limitación de algunos estudios es el nivel de agregación que consideran. En el de Runge y otros (1997) se utilizó la Clasificación Industrial Internacional Uniforme de todas las actividades económicas (CIIU) a nivel de dos dígitos, con categorías tan amplias como productos no metálicos y productos de metal. En los estudios sobre Argentina de Chudnovsky, Cap y otros (1999), aquellos sobre Brasil de Ferraz y Young (1999) y aquellos sobre América Latina y sobre México de Schatan (1999 y 2000), se usó la CIIU a nivel de tres dígitos, en la que las industrias manufactureras se subdividen en 28 sectores. Incluso a este nivel no se distingue entre el curtido y el acabado del cuero (3231) y la fabricación de productos de cuero (3233), aunque la primera actividad implica una emisión tóxica por cada millón de dólares de producto más de 200 veces superior a la segunda (Hettige, Martin y otros, 1995, cuadro 5.5). ${ }^{13}$ En el presente estudio se aminora este problema, ya que se estima la contaminación a partir de información comercial de 82 industrias clasificadas a nivel de cuatro dígitos de la CIIU.

\section{IV}

\section{Un enfoque alternativo del comercio}

\section{y la contaminación}

\section{La contaminación como factor de producción}

Teniendo en cuenta las limitaciones señaladas, en el presente artículo se adopta un enfoque diferente para analizar el efecto de la apertura en la contaminación industrial. El punto de partida de este enfoque es la consideración de las emisiones como un insumo del proceso productivo. Aunque a menudo las emisiones se conciben como un resultado de ese proceso, reflejan el hecho de que se hayan usado recursos ambientales en la producción (Rauscher, 1997). Por lo tanto, puede pensarse que los bienes comercializados "contienen" cierta cantidad de recursos ambientales. ${ }^{14}$ Según este enfoque, los intercambios comerciales se traducen en transferencias internacionales de recursos ambientales. Si las exportaciones de un país producen más contaminación que sus importaciones, el país es un exportador neto de recursos ambientales; en cambio, si se especializa en productos relativamente "limpios" es un importador de recursos ambientales.

\footnotetext{
${ }^{13}$ Este problema no es tan serio en los estudios que emplean la CUCI a nivel de tres dígitos.

${ }^{14}$ Lee y Roland-Holst (1993), que utilizaron el concepto de comercio de contaminantes incorporados (embodied effluent trade), adoptaron un enfoque similar.
}

Esta conceptualización de la contaminación es paralela a la de otros insumos — como capital humano, mano de obra y capital — en la literatura sobre "contenido factorial del comercio". El enfoque citado se ha empleado para comprobar el teorema de HeckscherOhlin y estimar el efecto del comercio sobre la demanda de factores de producción. Su origen se remonta al célebre estudio de Leontief sobre el comercio de los Estados Unidos que dio origen a la "paradoja de Leontief".

\section{Modelo general de contenido factorial}

Según el modelo general de contenido factorial, los coeficientes de contenido factorial de las exportaciones $i$ de cada país se estiman de la siguiente forma: ${ }^{15}$

$$
\mathrm{z}_{\mathrm{xi}}=A_{i} \mathrm{x}_{\mathrm{I}}
$$

donde $z_{x i}$ es un vector $(q \times 1)$ de la cantidad de factor por cada millón de dólares de exportaciones, A es una matriz de coeficientes ( $q \times r)$ de cada factor empleado por millón de dólares de producto en cada uno de

15 Éste es el razonamiento de Wood (1994, pp. 67 y 68). 
los sectores manufactureros del país, y $x$ es un vector $(r \times 1)$ de la participación sectorial en las exportaciones de manufacturas del país. Por otra parte, el contenido factorial de las importaciones puede calcularse como el promedio ponderado de los coeficientes de factores incorporados por sector $z_{m i}$ en las importaciones. Un factor es utilizado en forma muy intensiva en las exportaciones de un país cuando $z_{x i}>z_{m i}$.

Una vez que se ha reconocido que la contaminación puede considerarse un insumo de recurso ambiental incorporado a la producción, resulta lógico emplear coeficientes de emisión por dólar de producción para calcular el contenido de recursos ambientales del comercio. El efecto del comercio en la utilización de los recursos ambientales de un país puede calcularse de manera análoga a la demanda de mano de obra. Por lo tanto, el efecto global en la contaminación es el siguiente:

$$
\mathrm{Z}_{\mathrm{i}}=\mathrm{X}_{\mathrm{i}}\left(z_{x i}-z_{m i}\right)
$$

donde $Z_{\mathrm{i}}$ es un vector de diferentes emisiones y $\mathrm{X}_{\mathrm{i}}$ el valor total (escalar) de las exportaciones de manufacturas del país $i$.

Para emplear este modelo en la comprobación de las teorías de contenido factorial del comercio internacional, o la estimación del efecto del comercio en la demanda de cada factor, hay que tener en cuenta lo siguiente: el tipo de coeficientes que se deben usar, si deben ser nacionales o internacionales y cómo manejar los desequilibrios comerciales.

Ante todo hay que decidir si se emplearán los insumos del factor por dólar de valor agregado o por dólar de producción. Las emisiones de una determinada industria o empresa suelen estar más directamente relacionadas con el valor agregado dentro de la industria o empresa que con la producción bruta. Por otro lado, el comercio se mide en términos de la producción bruta, no del valor agregado, salvo a menudo en el caso de las zonas francas de exportación. Esto plantea un dilema, ya que, como señala Wood (1994): si se emplea la producción bruta los efectos sobre la demanda se desdibujan; pero si se emplea el valor agregado la relación con las corrientes de intercambio comercial es menos evidente.

En la práctica, se tiende a emplear coeficientes calculados según la producción bruta.

También se deberá optar entre los coeficientes correspondientes al país estudiado o a su socio comercial. Se trata de una decisión importante, ya que las diferencias en cuanto al grado de rigor de las norma- tivas entrañan considerables desigualdades en las emisiones por unidad de producción entre los países desarrollados y menos desarrollados. La elección adecuada de los coeficientes depende del propósito que se persiga. Si lo que interesa medir es el efecto del comercio en lo que respecta a la carga real de contaminación en un país, sería aconsejable aplicar los coeficientes nacionales a las exportaciones e importaciones, suponiendo que las importaciones se podrían producir dentro del país con el mismo nivel de contaminación por unidad de producción exhibido por las industrias que compiten con las importaciones.

Sin embargo, el objetivo del presente artículo no es ése, sino determinar si la apertura ha llevado a una especialización mayor en los países de América Latina en industrias consideradas muy contaminantes en el hemisferio norte. Por tal motivo, resulta adecuado emplear los coeficientes de los países desarrollados como indicadores de la intensidad de contaminación. Esto representa una ventaja, dado que la fuente más importante de información detallada sobre emisiones por dólar son los datos de los Estados Unidos, y esos son los coeficientes que se emplean. ${ }^{16}$

Por último, es poco probable que el comercio de manufacturas de un determinado país esté en equilibrio, lo que obliga a decidir si se debe o no tomar en cuenta este hecho al estimar los efectos en la demanda de factores. En los últimos años, en los países de América Latina la balanza comercial ha sufrido acentuadas oscilaciones, lo que tiende a distorsionar la evolución de las transferencias de recursos ambientales de año en año. El enfoque tradicional es el de considerar un comercio balanceado, lo que está implícito en la ecuación [2], donde se aplican coeficientes ponderados de emisión de las importaciones y exportaciones al valor de las exportaciones (Wood, 1994).

\section{Formulación de las hipótesis}

Los paraísos de contaminadores en América Latina pueden examinarse desde varios puntos de vista. La

\footnotetext{
${ }^{16}$ Esta es otra razón por la que este análisis no puede emplearse para medir el efecto de la apertura en la contaminación en un país en proceso de liberalización. Dado que la normativa ambiental es más estricta en los Estados Unidos que en América Latina, el uso de los coeficientes estadounidenses lleva a subestimar la carga de contaminación real asociada a las exportaciones de América Latina. Sin embargo, si se supone que el nivel relativo de contaminación vinculado a las industrias no difiere demasiado de un país a otro, los cálculos indican la dirección del cambio en la contaminación real.
} 
primera pregunta que se plantea en este contexto es si la intensidad de contaminación de las exportaciones en general supera a la de las importaciones. Si lo hace, habría una transferencia neta de recursos ambientales de América Latina al resto del mundo. Esto significaría que América Latina tiene una ventaja comparativa revelada en industrias altamente contaminantes. Sin embargo, puesto que la estructura real de los intercambios comerciales refleja las restricciones al comercio existente, esto no significa que tal ventaja comparativa se mantendría necesariamente en condiciones de libre comercio.

Una segunda interrogante es si la apertura comercial trae aparejada una mayor especialización en industrias muy contaminantes. Para determinarlo, se puede examinar la variación de la intensidad de contaminación relativa de las exportaciones y de las importaciones, a partir de la apertura. Si esta razón muestra un aumento, se puede concluir que la hipótesis de paraísos de contaminadores, en su sentido amplio de desplazamiento de la producción contaminante, es válida. Como ya se indicó, en estudios anteriores sobre contaminación y comercio en América Latina sólo se examinó la composición de las exportaciones. Esto puede conducir a conclusiones equívocas, porque hay datos que demuestran que en los últimos años el comercio mundial de productos de industrias relativamente contaminantes ha crecido a un ritmo menor que el de productos de las industrias menos contaminantes (Xu, 1999, cuadro 1). Por lo tanto, para evaluar cualquier hipótesis relativa a la especialización es fundamental considerar las importaciones junto con las exportaciones, enfoque que se adopta en los estudios tradicionales sobre el contenido factorial del comercio internacional. En los escasos estudios en que se ha considerado el contenido factorial en relación con cuestiones ambientales también se tuvieron en cuenta tanto las exportaciones como las importaciones (Kalt, 1988; Robison, 1988; Lee y Roland-Holst, 1993).

La tercera pregunta que se plantea es si la apertura comercial propicia la disminución o el aumento de la contaminación en los países de América Latina. A primera vista, esto parecería ser una simple variación del enfoque descrito en el párrafo anterior, pero en realidad no es así. Para aclarar la situación ayudaría establecer una analogía con los efectos de dicha apertura en lo que respecta al empleo. Si las exportaciones implican un uso más intensivo de mano de obra que la producción que compite con las importaciones, un aumento paralelo de las exportaciones e importaciones se traducirá en un incremento del empleo, incluso si no aumenta el contenido medio de mano de obra en las exportaciones. Asimismo, aunque en un país no se incremente la especialización en industrias contaminantes, la contaminación total puede aumentar de todos modos. tesis:

Lo indicado permite formular las siguientes hipó-

- Hipótesis 1: Los países de América Latina tenderán a especializarse en industrias relativamente contaminantes, de manera que en un momento dado $z_{x i}>z_{m i}$

- Hipótesis 2: A raíz de la liberalización, en los países de América Latina la especialización en industrias contaminantes tenderá a aumentar, de manera que el $z_{x i} / z_{m i}$ posterior a la liberalización superará al de la etapa anterior.

- Hipótesis 3: A raíz de la liberalización, el efecto combinado del incremento del volumen (efecto de escala) y de la composición cambiante del comercio elevará el grado de contaminación a nivel nacional en América Latina.

\section{V}

\section{Apertura del comercio y paraísos de contaminadores en Argentina, Brasil y México}

\section{Datos}

Para poner a prueba estas hipótesis se requiere información sobre los coeficientes de emisión y sobre las exportaciones e importaciones. En la mayoría de los estudios realizados se ha utilizado un solo indicador, generalmente relacionado con las emisiones tóxicas. Sin embargo, como se ha dicho antes, no existe necesariamente una correlación entre todos los indicadores del grado de contaminación, por lo que el empleo de un indicador único puede llevar a conclusiones erróneas (Hettige, Martin y otros, 1995). Por consiguiente, 
en este análisis se optó por tomar en cuenta el espectro de contaminantes más amplio posible. Los coeficientes elegidos corresponden al International Pollution Projection System (IPPS), ${ }^{17}$ y fueron calculados por el Banco Mundial a partir de datos de los Estados Unidos que cuantifican una amplia gama de contaminantes por dólar de producción, valor agregado o trabajador. ${ }^{18}$

Entre ellos se hallan las sustancias tóxicas, los metales tóxicos, seis contaminantes atmosféricos convencionales (dióxido de azufre, dióxido de nitrógeno, monóxido de carbono, compuestos orgánicos volátiles, material particulado respirable y total de partículas en suspensión) y dos contaminantes del agua (la demanda bioquímica de oxígeno y el total de sólidos en suspensión). En el presente estudio se optó por los coeficientes basados en la producción ya que, como se ha comentado, las exportaciones y las importaciones se miden en términos de producción bruta más que de valor agregado.

Estos coeficientes se aplicaron a las cifras de importaciones y exportaciones de manufacturas proporcionadas por la CEPAL, reclasificadas a nivel de cuatro dígitos de la cucr. Los datos de comercio se limitaron a los productos manufacturados porque la hipótesis de paraísos de contaminadores se aplica sobre todo a actividades que no están "atadas" a un lugar geográfico determinado. Es menos factible que las diferencias en la normativa ambiental afecten otros sectores, como la minería y la agricultura, cuya movilidad geográfica es menor. ${ }^{19}$

\section{Resultados}

La primera interrogante que debe considerarse es si estos tres países de América Latina se especializan o no en industrias relativamente contaminantes y, por ende, si son exportadores netos de recursos ambientales. Como se indicó, esto se puede determinar mediante

\footnotetext{
${ }^{17}$ El uso de coeficientes derivados de los datos de contaminación en los Estados Unidos es coherente con el propósito de evaluar si en los países de América Latina ha aumentado la especialización en industrias consideradas muy contaminantes en los países desarrollados.

${ }^{18}$ En Hettige, Martin y otros (1995) se pueden encontrar detalles del IPPSS. Los coeficientes se pueden consultar en "Nuevas ideas en reglamentación de la contaminación", en la página de Internet del Banco Mundial (http://www.worldbank.org/html/prdei/ipps/ ippshome.html).

${ }^{19}$ En Warhurst y Hughes-Witcomb (2001) se considera la hipótesis de los paraísos de contaminadores en la minería en América Latina y se llega a la conclusión de que no es válida.
}

la comparación de la intensidad de contaminación media por dólar de exportaciones con la correspondiente por dólar de importaciones. En el cuadro 2 se muestra la relación entre la contaminación por dólar de exportaciones y por dólar de importaciones para cada contaminante, tanto al inicio del proceso de apertura en cada país como en 1996, último año para el que se dispone de datos de comercio.

En los datos de 1996 se aprecia un contraste muy marcado entre Argentina y Brasil, por una parte, y México, por la otra. En los dos países sudamericanos las exportaciones contaminan más que las importaciones en ocho de los diez contaminantes considerados; las excepciones son las sustancias tóxicas y la demanda bioquímica de oxígeno en el caso argentino y las sustancias tóxicas y los compuestos orgánicos volátiles en el brasileño. Por el contrario, en México la intensidad de contaminación de las importaciones es superior en todos los indicadores, salvo en el caso del material particulado respirable (PM10). Por consiguiente, los casos de Argentina y Brasil apoyan ampliamente la primera hipótesis, es decir, que los países de América Latina son exportadores netos de recursos ambientales, pero el caso de México no.

En el cuadro 2 figuran también datos del cociente entre la intensidad de contaminación de las exportaciones y de las importaciones antes de la apertura. En el caso de Argentina y Brasil, estas cifras corresponden a 1990, mientras que en el caso de México, en que la liberalización se inició antes, se tomó 1985 como punto de comparación. El contraste entre los países de América del Sur y México es también pronunciado en lo que respecta a la evolución de las intensidades de contaminación relativas de las exportaciones y de las importaciones tras el inicio de la apertura de sus economías. A lo largo del período, en Argentina y Brasil se elevaron ocho de los diez indicadores, mientras que en México, todos salvo uno disminuyeron. Por lo tanto, los casos de Argentina y Brasil apoyan ampliamente la segunda hipótesis, pero el caso de México la refuta de plano. Hay que señalar que, antes de la apertura, la situación en México era similar a la argentina y la brasileña, dado que a mediados del decenio de 1980 las exportaciones tendían a contaminar más que las importaciones con la mayoría de los contaminantes.

La tercera hipótesis se refiere al efecto de la apertura sobre las emisiones en los países de América Latina, que puede concebirse como el efecto combinado de la escala y el cambio de la composición del comercio. Si en un primer momento las exportaciones contaminan más que las importaciones, un incremen- 


\begin{tabular}{|c|c|c|c|c|c|c|}
\hline & \multicolumn{2}{|c|}{ Argentina } & \multicolumn{2}{|c|}{ Brasil } & \multicolumn{2}{|c|}{ México } \\
\hline & 1990 & 1996 & 1990 & 1996 & 1985 & 1996 \\
\hline Sustancias tóxicas & 0,54 & 0,86 & 0,70 & 0,88 & 0,88 & 0,67 \\
\hline Metales & 1,21 & 1,27 & 2,17 & 2,68 & 1,31 & 0,99 \\
\hline Dióxido de azufre $\left(\mathrm{SO}_{2}\right)$ & 1,47 & 1,61 & 1,77 & 1,87 & 1,78 & 0,83 \\
\hline Dióxido de nitrógeno $\left(\mathrm{NO}_{2}\right)$ & 1,02 & 1,19 & 1,29 & 1,31 & 1,42 & 0,67 \\
\hline Monóxido de carbono (CO) & 1,03 & 1,03 & 2,03 & 2,14 & 1,16 & 0,74 \\
\hline Compuestos orgánicos volátiles & 0,90 & 1,13 & 0,97 & 0,94 & 1,43 & 0,78 \\
\hline Material particulado respirable (PM10) & 4,69 & 6,79 & 4,50 & 4,08 & 3,48 & 1,62 \\
\hline Total de partículas en suspensión & 3,19 & 4,29 & 2,56 & 2,88 & 1,80 & 0,96 \\
\hline Demanda bioquímica de oxígeno & 0,70 & 0,80 & 1,08 & 1,37 & 0,65 & 0,42 \\
\hline Total de sólidos en suspensión & 1,66 & 1,55 & 4,39 & 4,89 & 0,74 & 0,88 \\
\hline
\end{tabular}

Fuente: Elaboración del autor en función de datos de comercio proporcionados por la CEPAL y de los coeficientes del International Pollution Projection System (IPPS).

to del comercio producirá un aumento neto de las emisiones, y el efecto de escala será positivo. ${ }^{20}$ Dado que al inicio de la liberalización ésta era la situación de la mayor parte de los indicadores en los tres países, y que en Argentina y Brasil los cambios en la composición han tendido a elevar la intensidad de contaminación relativa, sería de esperar que también hayan aumentado las emisiones totales en ambos países. En México, donde las exportaciones se fueron haciendo relativamente menos contaminantes con el transcurso del tiempo, el efecto combinado de la escala y la composición es ambiguo.

En el cuadro 3 figura la variación neta de las emisiones de cada contaminante desde el inicio de la apertura (en 1985 en México y en 1990 en Argentina y Brasil) hasta 1996. Estas cifras se establecieron mediante la aplicación de los coeficientes de emisión de cuatro dígitos del Sistema para proyectar la contaminación industrial a la estructura de las exportaciones e importaciones, en el año base y en 1996, y el cálculo de la variación entre ambas fechas. Como ya se indicó, para que los cambios anuales de la balanza comercial de manufacturas no afectaran los resultados, el cálculo se basó en el supuesto de que la importación y la exportación total de manufacturas eran equi-

\footnotetext{
${ }^{20}$ Se debe tener en cuenta que, cuando se considera la producción, la situación es diferente. En ese caso, el efecto de escala es positivo por definición, ya que si el resto de las condiciones no varía, un aumento de la producción no puede reducir la contaminación. Pero cuando se trata del comercio, es decir, cuando las exportaciones e importaciones aumentan, el efecto de la escala en el aumento o la disminución de la contaminación dependerá de si las exportaciones generan más emisiones que los sustitutos de las importaciones.
}

parables. En otras palabras, la variación de las emisiones se calculó de acuerdo a la siguiente fórmula:

$$
\mathrm{Z}_{\mathrm{i}}^{\prime}-\mathrm{Z}_{\mathrm{i}}=\mathrm{X}_{\mathrm{i}}^{\prime}\left(z_{x i}^{\prime}-z_{m i}^{\prime}\right)-\mathrm{X}_{\mathrm{i}}\left(z_{x i}-z_{m i}\right)
$$

donde ' indica la cifra correspondiente al año final, y los símbolos sin' corresponden al año base.

El contraste entre Argentina y Brasil, por una parte, y México por otra, no podría ser más marcado. Tras la apertura, en los dos países sudamericanos se registró un aumento de las emisiones locales de nueve de los diez contaminantes. ${ }^{21}$ En México, salvo la emisión de material particulado respirable, todas disminuyeron. Como era previsible, en Argentina y Brasil el efecto combinado de la expansión y el cambio en la composición del comercio provocó un aumento de la contaminación. En México, el efecto del cambio en la composición, orientado a incluir industrias menos contaminantes, compensó con creces el aumento del comercio. Por lo tanto, el caso mexicano es un ejemplo del cambio anunciado por quienes sostienen que el libre comercio no arrojará más que ventajas.

\section{Interpretación}

¿A qué se debe el sostenido contraste, que se pone de manifiesto en este análisis, entre México y los dos países sudamericanos considerados? Como se indicó, algunos autores predicen que en los países en desarrollo el

\footnotetext{
${ }^{21}$ Esto se traduce en un mayor superávit o en un menor déficit de recursos ambientales exportados.
} 
CUADRO 3

Argentina, Brasil y México: Cambios en las emisiones netas asociadas al comercio tras la apertura

(En miles de libras)

\begin{tabular}{lcrc}
\hline & Argentina & Mrasil & México \\
& $(1990-1996)$ & $(1995-1996)$ \\
\hline Sustancias tóxicas & 23112 & 23571 & -60102 \\
Metales & 179 & 5598 & -1504 \\
Dióxido de azufre $\left(\mathrm{SO}_{2}\right)$ & 7804 & 33428 & -65843 \\
Dióxido de nitrógeno $\left(\mathrm{NO}_{2}\right)$ & 5309 & 7880 & -50347 \\
Monóxido de carbono $(\mathrm{CO})$ & 43 & 29974 & -56377 \\
Compuestos orgánicos volátiles & 5345 & -2268 & -27862 \\
Material particulado respirable (PM10) & 8222 & 6420 & 3 \\
Total de partículas en suspensión & 12623 & -7521 \\
Demanda bioquímica de oxígeno & 745 & 17749 & -19389 \\
Total de sólidos en suspensión & -9670 & 8226 & -38600 \\
\hline
\end{tabular}

Fuente: Cálculos del autor en función de los coeficientes del International Pollution Projection System (IPPS) y con datos de la CEPAL sobre el comercio.

grado de contaminación emanada de la industria disminuiría con la apertura comercial, entre otras razones, porque creen que las políticas proteccionistas de sustitución de importaciones tienden a favorecer a las "industrias sucias" y que su supresión eliminaría este "sesgo sucio". ¿En qué medida sucedió esto en los tres países de América Latina considerados, en el período anterior a la reciente apertura?

Para responder a esta pregunta se analizó la estructura de protección en Argentina, Brasil y México antes de la apertura. Se reunieron estimaciones sectoriales pertinentes de la tasa efectiva de protección de Brasil en 1973 (Coes, 1991, cuadro 4.1), de Argentina en 1980 (Cavallo y Cottani, 1991, cuadro 3.19) y de México en 1979 (Ten Kate y Mateo Venturini, 1989, cuadro 4). Sobre la base de estos datos, se clasificaron las industrias según el mayor o menor nivel de protección efectiva. ${ }^{22}$ En el caso de Brasil y México, se consideró que el nivel de protección más alto correspondía a una tasa efectiva superior al 50\%, mientras que en el caso argentino, caracterizado por un nivel de protección general más elevado, el límite fue del $75 \%$.

Tras la clasificación, se pudo calcular la intensidad de contaminación media de las industrias con mayor y con menor tasa efectiva de protección y obtener la relación entre la intensidad de contaminación generada por uno y otro grupo. Si la protección tiene un

\footnotetext{
${ }^{22}$ Debido a la falta de estimaciones sobre la protección efectiva se omitieron algunas industrias. En los casos en que las estimaciones disponibles correspondían al nivel de dos dígitos, se supuso que las industrias del nivel de tres dígitos eran objeto de la misma protección que la categoría de dos dígitos a la que pertenecían.
}

"sesgo sucio", es decir, tiende a proteger las industrias más contaminantes, el cociente es superior a 1. Contrariamente a lo que se podría esperar, en Argentina y Brasil el sesgo era el opuesto: las industrias más protegidas tenían emisiones relativamente bajas de la mayoría de los contaminantes (cuadro 4). Sólo el caso mexicano se atuvo a lo previsto: intensidad de contaminación relativamente alta de sus industrias más protegidas, salvo en el total de sólidos en suspensión y de material particulado.

Esta estructura de protección podría explicar los diferentes resultados en los tres países tras la apertura. En México, donde antes de la apertura las industrias "sucias" eran objeto de una gran protección, los cambios en la política comercial provocaron una contracción de estas industrias en relación con otras menos contaminantes y, por lo tanto, una reducción de las emisiones. ${ }^{23}$ En Argentina y Brasil, donde la estructura de protección no tenía un "sesgo sucio", las exportaciones siguieron siendo más contaminantes que las importaciones, tal como lo previsto según la hipótesis de "paraísos de contaminadores".

Otro factor que puede explicar en parte los efectos diferentes de la apertura en la intensidad de contaminación en los países examinados es la adopción por México — a principios del decenio de 1990 y mientras

\footnotetext{
${ }^{23}$ Resulta significativo que el material particulado respirable sea el único contaminante cuya emisión aumentó tras los cambios de las corrientes comerciales registrados de 1985 a 1996 (véase el cuadro 3). En el cuadro 4 se aprecia que el nivel de emisión por unidad de producto de este contaminante, contrariamente al resto, alcanzaba cifras mucho más altas en las industrias menos protegidas.
} 

industrias con mayor y menor tasa de protección efectiva

\begin{tabular}{|c|c|c|c|}
\hline & $\begin{array}{c}\text { Argentina } \\
1980\end{array}$ & $\begin{array}{c}\text { Brasil } \\
1973\end{array}$ & $\begin{array}{c}\text { México } \\
1979\end{array}$ \\
\hline Total de sustancias tóxicas & 0,32 & 0,17 & 1,42 \\
\hline Total de metales & 1,11 & 0,05 & 1,60 \\
\hline Demanda bioquímica de oxígeno & 0,81 & 1,32 & 1,34 \\
\hline Total de sólidos en suspensión & 0,30 & 0,01 & 0,41 \\
\hline Dióxido de nitrógeno $\left(\mathrm{NO}_{2}\right)$ & 0,13 & 0,39 & 2,30 \\
\hline Dióxido de azufre $\left(\mathrm{SO}_{2}\right)$ & 0,24 & 0,19 & 3,64 \\
\hline Monóxido de carbono (CO) & 0,23 & 0,11 & 2,01 \\
\hline Total de partículas & 0,21 & 0,44 & 0,98 \\
\hline Compuestos orgánicos volátiles & 0,15 & 0,63 & 3,27 \\
\hline
\end{tabular}

Fuente: Cálculos del autor en función de los coeficientes del International Pollution Projectios System (IPPS) y datos de la Organización de las Naciones Unidas para el Desarrollo Industrial (ONUDI).

se negociaba el TLC de América del Norte- de una normativa ambiental más exigente, sobre todo en lo que respecta a su control y cumplimiento (Poder Ejecutivo Federal, 1996). Esta medida respondió a temores de ciudadanos estadounidenses de que bajo el régimen de libre comercio México se transformara en un paraíso de contaminación, temores que amenazaban con impedir el acuerdo (Hogenboom, 1998, cap. 6). La normativa ambiental más estricta habría tendido a reducir la ventaja comparativa en materia de industrias altamente contaminantes que tenía México en la década de 1980.

\section{VI}

\section{Conclusión}

El presente artículo partió con el propósito de determinar si lo sucedido en los principales países de América Latina tras los inicios de la apertura comercial confirmaba los temores de los ambientalistas sobre el efecto de este proceso en la contaminación industrial. Se ha centrado en particular en la hipótesis de paraísos de contaminadores, entendida como la tendencia de los países cuya normativa ambiental es menos estricta a especializarse en industrias muy contaminantes.

En Argentina y Brasil se observa que la apertura ha ido asociada a un cambio hacia una mayor especialización en industrias contaminantes y a una tendencia ascendente del nivel de contaminación en el ámbito local. México exhibe la tendencia contraria, orientada a la especialización en sectores menos contaminantes y a la reducción de la contaminación local. Se ha sugerido aquí que esto podría reflejar diferencias en la estructura de protección anterior a la apertura comercial, es decir, a la mayor protección de las industrias "sucias" que habría habido en México. También se ha señalado que en este país, en forma paralela a la apertura, se adoptó una normativa ambiental más estricta, en parte con miras a calmar los temores surgidos en los Estados Unidos ante posibles paraísos de contaminadores y éxodo industrial.

Antes de concluir, es imprescindible formular algunas salvedades. Aunque el análisis se haya basado en datos mucho más desagregados que cualquier otro estudio previo sobre comercio y medio ambiente en América Latina (a nivel de cuatro dígitos de la ciIU), tal desagregación puede seguir siendo insuficiente para captar algunos de los procesos que se están registrando en cada rama de actividad industrial. Es el caso de las curtiembres en Brasil, en las que se ha detectado un aumento de la especialización en el curtido al cromo húmedo, orientado al mercado de exportación (Odegard, 2000). Este proceso es la etapa más contaminante del curtido, e implica escaso valor agregado. En las curtiembres de los países desarrollados se está recurriendo a la contratación externa del curtido 
al cromo húmedo, mientras que a nivel nacional se utilizan procesos menos contaminantes y con mayor valor agregado. Se trata entonces de un caso claro de "transferencia de contaminación" a Brasil en esta actividad. Sin embargo, esto es indetectable a través de los datos agregados, incluso al nivel de cuatro dígitos de la ciıu, en el cual la categoría 3231 (curtido y acabado del cuero) abarca la totalidad del procesamiento del cuero.

La segunda salvedad se refiere a aquello que escapa a los objetivos del presente artículo. En éste no se evalúa de manera exhaustiva si la apertura ha entrañado un aumento de la contaminación en América Latina. Un análisis de esa índole obligaría a considerar no sólo los efectos de escala y composición derivados del incremento del comercio en lo que respecta a la contaminación, sino también los efectos de la apertura en términos de proceso (Grossman y Krueger, 1992). Los datos empleados en el presente artículo, que se basa en derivados de coeficientes de emisión preestablecidos, impiden de antemano un análisis de este último tipo. Al pasar revista en este artículo a las consecuencias en materia de contaminación a nivel nacional en América Latina, se ignoraron estos efectos. Sin embargo, dado que su objetivo principal es considerar la distribución de las actividades relativamente contaminantes, lo dicho no invalida el examen de la hipótesis de paraísos de contaminadores.

Una evaluación exhaustiva de los efectos relativos de la apertura sobre la contaminación en los países debería considerar otros vínculos posibles entre el comercio y la contaminación. Como aspecto positivo, se señaló que producir para los mercados de exportación se traduce en presiones sobre las empresas para que reduzcan la contaminación y mejoren sus sistemas de gestión ambiental. También se indicó que la apertura comercial permite el acceso más amplio a tecnologías importadas y procesos más limpios. En sentido contrario, el aumento de la competencia resultante de la apertura hace que en las empresas disminuya la capacidad y la voluntad de internalizar los costos ambientales, y atenta contra los esfuerzos orientados a redu- cir la contaminación. El papel de las inversiones internacionales suscita debates similares. Lamentablemente, este artículo no es lo suficientemente extenso como para ocuparse de esos temas. ${ }^{24}$

Una razón adicional por la que este artículo no puede ser tomado como una evaluación exhaustiva de los efectos ambientales de la apertura comercial es que no tienen en cuenta las consecuencias de ella en la política ambiental. Para sus detractores, esa apertura conduce a una carrera cuesta abajo en materia de estándares ambientales, ya que las normas ambientales menos exigentes significan una ventaja competitiva para las empresas. Para quienes sustentan una posición más moderada, el incremento de la apertura ha paralizado la evolución de la normativa ambiental. Un análisis exhaustivo del efecto de la apertura comercial en América Latina debería, por lo menos, determinar si esto sucede efectivamente y si, por ende, incide de manera potencial e indirecta en el aumento de la contaminación.

En el presente artículo se aplicó una metodología basada en el contenido factorial del comercio para analizar la intensidad relativa de contaminación de las exportaciones con respecto a la de las importaciones en los países semiindustrializados de América Latina. En este análisis se reveló que, a principios de la década de 1990, tanto Argentina como Brasil y México se especializaban en industrias relativamente "sucias". Sin embargo, desde entonces en México se ha registrado un proceso opuesto al de los países del Cono Sur. Estos últimos se han desplazado crecientemente hacia industrias más contaminantes, mientras que México ha aumentado su ventaja comparativa en industrias menos contaminantes. El pronóstico de la hipótesis de paraísos de contaminadores no se cumplió en el caso de México, debido al pronunciado sesgo proteccionista en favor de las "industrias sucias" anterior a la apertura y al cumplimiento más estricto de la normativa ambiental en la década de 1990.

(Traducido del inglés)

\footnotetext{
${ }^{24}$ En Jenkins (2000) se examinan datos sobre estos temas.
} 
Agosin, M. y R. Ffrench-Davis (1993): La liberalización comercial en América Latina, Revista de la CEPAL, No 50, LC/G.1767-P, Santiago de Chile, agosto.

Antweiler, W., B. Copeland y S. Taylor (1998): Is Free Trade Good for the Environment?, Discussion Paper, $\mathrm{N}^{\mathrm{o}}$ 98-11, Vancouver, Departamento de Economía, University of British Columbia.

Aroche, F. (2000): Reformas estructurales y composición de las emisiones contaminantes industriales. Resultados para México, serie Medio ambiente y desarrollo, $\mathrm{N}^{\circ}$ 24, LC/L.1333-P, Santiago de Chile, CEPAL. Publicación de las Naciones Unidas, $\mathrm{N}^{\mathrm{o}}$ de venta: S.00.II.G.42.

Basualdo, E., E. Lifschitz y E. Roca (1988): Las empresas multinacionales en la ocupación industrial en la Argentina, 19731983, Ginebra, Oficina Internacional del Trabajo.

Baumol, W. y W. Oates (1988): The Theory of Environmental Policy, Nueva York, Cambridge University Press.

Beghin, J. y M. Potier (1997): Effects of trade liberalisation on the environment in the manufacturing sector, The World Economy, vol. 20, No 4, Oxford, Blackwell Publishers, junio.

BID (Banco Interamericano de Desarrollo) (1997): Progreso económico y social en América Latina. Informe 1997, Washington D.C.

Birdsall, N. y D. Wheeler (1992): Trade policy and industrial pollution in Latin America: Where are the pollution havens?, en P. Low (comp.), International Trade and the Environment, World Bank Discussion Papers, No 159, Washington, D.C., Banco Mundial.

Burki, S. y G. Perry (1997): The Long March: A Reform Agenda for Latin America and the Caribbean in the Next Decade, Washington, D.C., Banco Mundial.

Cavallo, D. y J. Cottani (1991): Argentina, en D. Papageorgiou, M. Michaely y S. Choksi (comps.), Liberalizing Foreign Trade, vol. 1, Cambridge, Massachusetts, Basil Blackwell.

CEPAL (Comisión Económica para América Latina) (2001): Anuario estadístico de América Latina y el Caribe, 2000, LC/g.2118-P, Santiago de Chile. Publicación de las Naciones Unidas, $N^{\circ}$ de venta S/E.00.II.G.01.

Chudnovsky, D y A. López (1997): Las estrategias de las empresas transnacionales en Argentina y Brasil: ¿qué hay de nuevo en los años noventa?, Documento de trabajo, $\mathrm{N}^{\circ} 23$, Buenos Aires, Centro de Investigaciones para la Transformación (CENIT).

Chudnovsky, D., F. Porta y otros (1996): Los límites de la apertura: liberalización, reestructuración productiva y medio ambiente, Buenos Aires, Centro de Investigaciones para la Transformación (CENIT)/Alianza Editorial.

Chudnovsky, D., E. Cap y otros (1999): Comercio internacional y desarrollo sustentable: la expansión de las exportaciones argentinas en los años 1990 y sus consecuencias ambientales, Documento de trabajo, $\mathrm{N}^{\circ} 25$, Buenos Aires, Centro de Investigaciones para la Transformación (CENIT).

Coes, D. (1991): Brazil, en D. Papageorgiou, M. Michaely y S. Choksi (comps.), Liberalizing Foreign Trade, vol. 1, Cambridge, Massachusetts, Basil Blackwell.

Copeland, B. y S. Taylor (1994): North-South trade and the environment, The Quarterly Journal of Economics, vol. 109, $\mathrm{N}^{\circ}$ 3, Massachusetts, The MIT Press, agosto.

Dean, J., (1992): Trade and the environment: a survey of the literature, en P. Low (comp.), International Trade and the Environment, World Bank Discussion Papers, No 159 , Washington, D.C., Banco Mundial.

Dijkstra, G. (1997): Trade Liberalisation and Industrial Development: Theory and Evidence from Latin America, Working Paper Series, No 255 , La Haya, Instituto de Estudios Sociales (ISS).

Edwards, S. (1995): Crisis and Reform in Latin America: From Despair to Hope, Washington, D.C., Banco Mundial.
Esty, D. (1994): Greening the GATT: Trade, Environment and the Future, Washington, D.C., Institute for International Economics.

Ferraz, C. y C. Young (1999): Trade Liberalization and Industrial Pollution in Brazil, serie Medio ambiente y desarrollo, $\mathrm{N}^{\circ} 23$, LC/L.1332-P, Santiago de Chile, CEPAL. Publicación de las Naciones Unidas, $\mathrm{N}^{\mathrm{o}}$ de venta: S.00.II.G.47.

Fritsch, W. y G. Franco (1991): Foreign Direct Investment in Brazil: Its Impact on Industrial Restructuring, París, Organización de Cooperación y Desarrollo Económicos (OCDE).

Grossman, G. y A. Krueger (1992): Environmental Impacts of a North American Free Trade Agreement, NBER Working Paper,

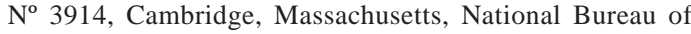
Economic Research.

Hettige, H., P. Martin y otros (1995): The Industrial Pollution Projection System, Policy Research Working Paper, No 1431 , Washington, D.C., Banco Mundial.

Hogenboom, B. (1998): Mexico and the NAFTA Environment Debate, Utrecht, International Books.

Jenkins, R. (2000): Globalisation, trade liberalisation and industrial pollution in Latin America, en R. Jenkins (comp.), Industry and Environment in Latin America, Londres, Routledge.

Jensen, V. (1996): The pollution haven hypothesis: some perspectives on theory and empirics, en S. Hansen, J. Hesselberg y H. Hveem, International Trade Regulation, National Development Strategies and the Environment: Towards Sustainable Development?, Occasional Paper, $\mathrm{N}^{\mathrm{o}}$ 2, Centre for Developmen and the Environment, Universidad de Oslo.

Jha, V., A. Markandya y R. Vossenaar (1999): Reconciling Trade and the Environment: Lessons from Case Studies in Developing Countries, Cheltenham, Edward Elgar.

Kalt, J. (1988): The impact of domestic environmental regulatory policies on U.S. international competitiveness, en M. Spence y H. Hazard (comps.), International Competitiveness, Cambridge, Massachusetts, Ballinger Publishing.

Lee, H. y D. Roland-Holst (1993): International Trade and the Transfer of Environmental Costs and Benefits, Technical Papers, No 91, París, Centro de Desarrollo de la Organización de Cooperación y Desarrollo Económicos (OCDE).

Low, P. (1992): Trade measures and environmental quality: the implications for Mexico's exports, en P. Low (comp.) International Trade and the Environment, World Bank Discussion Papers, $\mathrm{N}^{\mathrm{o}}$ 159, Washington, D.C., Banco Mundial.

Mabey, N. y R. McNally (1999): Foreign Direct Investment and the Environment: From Pollution Havens to Sustainable Development, Londres, WWF-UK.

Mani, M. y D. Wheeler (1999): In search of pollution havens? Dirty industry in the world economy, 1960-1995, P. Fredriksson (comp.), Trade, Global Policy and The Environment, World Bank Discussion Paper, $N^{\circ}$ 402, Washington, D.C., Banco Mundial.

MMA (Ministerio del Medio Ambiente) (2001), Comércio e meio ambiente: uma agenda positiva para o desenvolvimento sustentável, Secretaria de Políticas para el Desarrollo Sustentable, documento preparado para la XIII reunión del Foro de Ministros de Medio Ambiente de América Latina y el Caribe [http://www.mma.gov.br/port/sds/c\&ma/corpo.html)].

Odegard, J.T. (2000): Economic liberalisation and the environment: a case study of the leather tanning industry in Brazil, en R. Jenkins (comp.), Industry and Environment in Latin America, Londres, Routledge.

Poder Ejecutivo Federal (1996): Programa de Medio Ambiente 19952000, México, D.F., Instituto Nacional de Ecología.

Rauscher, M. (1997): International Trade, Factor Movements, and the Environment, Oxford, Clarendon Press. 
Robison, D. (1988): Industrial pollution abatement: the impact on balance of trade, Canadian Journal of Economics, vol. 21, $\mathrm{N}^{\circ} 1$.

Rock, D. (1996): Pollution intensity of GDP and trade policy: can the World Bank be wrong?, World Development, vol. 24, № 3.

Rodrik, D. (1994): King Kong meets Godzilla: The World Bank and the East Asian miracle, en A. Fishlow y otros (comps.), Miracle or Design? Lessons from the East Asian Experience, Washington, D.C., Consejo de Desarrollo de Ultramar.

Ros, J., J. Draisma y otros (1996): Prospects for growth and the environment in Mexico in the 1990s, World Development, vol. 24, $\mathrm{N}^{\mathrm{o}}$ 2, febrero.

Runge, C.F. y otros (1997): Sustainable Trade Expansion in Latin America and the Caribbean: Analysis and Assessment, Washington, D.C., Instituto de los Recursos Mundiales.

Schaper, M. (1999): Impactos ambientales de los cambios en la estructura exportadora de nueve países de América Latina y el Caribe: 1980-1995, serie Medio ambiente y desarrollo, $\mathrm{N}^{\circ}$ 19, LC/L.1241-P, Santiago de Chile, CEPAL. Publicación de las Naciones Unidas, $\mathrm{N}^{\mathrm{o}}$ de venta: S.99.II.G.44.

Schatan, C. (1999): Contaminación industrial en los países latinoamericanos pre y post reformas económicas, serie Medio ambiente y desarrollo, No 22, LC/L.1331-P, Santiago de Chile, CEPAL. Publicación de las Naciones Unidas, $\mathrm{N}^{\circ}$ de venta: S.00.II.G.46.

(2000): Mexico's Manufacturing Exports and the Environment under NAFTA, documento preparado para el North American Symposium on Understanding the Linkages between Trade and Environment, Commission for Environmental Cooperation [www.cec.org/programs_projects/trade_environ_econ/pdfs/ Schatan.pdf)].

Stallings, B. y W. Peres (2000): Crecimiento, empleo y equidad: el impacto de las reformas económicas en América Latina y el Caribe
Santiago de Chile, Fondo de Cultura Económica (FCE)/CEPAL.

Ten Kate, A. (1993): Industrial Development and the Environment in Mexico, México, D.F., Secretaría de Comercio y Fomento Industrial

Ten Kate, A. y F. de Mateo Venturini (1989): Apertura comercial y estructura de la protección en México, Comercio exterior, vol. $39, \mathrm{~N}^{\circ} 6$.

UNCTAD (Conferencia de las Naciones Unidas sobre Comercio y Desarrollo) (2002), World Investment Report, 2000 Transnational Corporations and Export Competitiveness, Nueva York, Naciones Unidas. Publicación de las Naciones Unidas, $\mathrm{N}^{\circ}$ de venta E.02.II.D.4.

Warhurst, A. y N. Hughes-Witcomb (2001): Mining and the environment in Latin America: the pollution haven hypothesis revisited, en R. Jenkins (comp.), Industry and Environment in Latin America, Londres, Routledge.

Wood, A. (1994): North-South Trade, Employment and Inequality: Changing Fortunes in a Skill-Driven World, Oxford, Clarendon Press.

Xu, X. (1999): Do stringent environmental regulations reduce the international competitiveness of environmentally sensitive goods? A global perspective, World Development, vol. 27, $\mathrm{N}^{\circ} 7$, julio.

Young, C. (1998): Industrial pollution and export-oriented policies in Brazil, Revista brasileira de economia, vol. 52, $\mathrm{N}^{\circ} 4$, Rio de Janeiro, octubre-diciembre.

(coord.) (1999): Abertura comercial, competitividade e poluição: o comportamento da indústria brasileira, Relatório de pesquisa CNPq 523607/96, Rio de Janeiro, Instituto de Economía.

Young, C., M. Lustosa y otros (2001): Comércio e meio ambiente, Relatório de Pesquisa presentado para la red Ipea, Rio de Janeiro, Instituto de Economía. 